\title{
Emergent Potentials in Consistent Higher Derivative $N=1$ Supergravity
}

\section{Fotis Farakos*}

Natl. Tech. U., Athens

E-mail: fotisfemail.ntua.gr

\author{
Alex Kehagias \\ Natl. Tech. U., Athens \\ E-mail: kehagias@central.ntua.gr
}

\begin{abstract}
By employing consistent supersymmetric higher derivative terms, we show that the supersymmetric theories may have a sector where the scalar potential does no longer have the conventional form. The theories under consideration contain consistent higher-derivative terms which do not give rise to instabilities and ghost states. The chiral auxiliaries are still not propagating and can be integrated out. Their elimination gives rise to emerging potentials even when there is not a superpotential to start with. This novel feature of higher derivative supersymmetric chiral models is also extended to vector multiplets both in global and local supersymmetry. In particular, in supergravity, the emerging potentials give rise always to a de Sitter vacuum signaling supersymmetry breaking.
\end{abstract}

Proceedings of the Corfu Summer Institute 2012

September 8-27, 2012

Corfu, Greece

\footnotetext{
* Speaker.
} 


\section{Introduction: Scalar Potentials in $N=1$ Superspace}

Supersymmetry is an extension of the Poincare spacetime symmetry with the inclusion of fermionic generators. It has various remarkable properties concerning phenomenological and theoretical aspects of particle physics. In particular, supersymmetry is one of the most appealing candidates for new physics. It has not been observed so far and thus, it should be broken at some high energy scale if it is realised at all. The central role on how supersymmetry is broken is played by the scalar potential of the supersymmetry breaking sector. Scalar potentials in supersymmetry and supergravity have been extensively studied for theories with up to two derivatives. Even though it is known that introducing higher derivatives will spoil the form of the scalar potential, the self-consistency of the theory protects it from unconventional non-supersymmetric vacua [1]. Our task here is to discuss how scalar potentials are modified when higher derivatives are introduced. However, the higher derivatives we are interested in, are those which do not introduce instabilities and/or ghost states. This is a known drawback of such kind of interactions, connected with the so-called Ostrogradski [2] instability in classical physics. We will see that such "safe" higher derivatives may consistently be introduced in supergravity and we will determine the form of the potential for the scalars of the theory they produce. We will also see that such potentias are sustained by background fluxes and have de Sitter vacua indicating that supersymmetry is broken.

In this work we are discussing the bosonic sector of supersymmetric interactions that belong to a specific class of higher derivative theories with the following two properties

1. they do not introduce ghost states

2. they introduce a scalar potential without a superpotential or gauging.

These theories involve chiral and vector multiplets.

In $N=1$ superspace there is a number of conventional methods to introduce a scalar potential for a chiral superfield. The superpotential is the most widely used, in which case one employes a holomorphic function of the chiral superfield and after integrating out the auxiliary sector, a scalar potential appears. More specificaly, the free Wess-Zumino Lagrangian is given by [3]

$$
L_{0}=A \partial^{2} \bar{A}+i \partial_{a} \bar{\psi}_{\dot{\alpha}} \bar{\sigma}^{a \dot{\alpha} \alpha} \psi_{\alpha}+F \bar{F} .
$$

It is straightforward to integrate out the auxiliary field via its equations of motion

$$
F=0
$$

which for the massless and free theory (1.1) vanishes, leading to

$$
L_{0}=A \partial^{2} \bar{A}+i \partial_{a} \bar{\psi}_{\dot{\alpha}} \bar{\sigma}^{a \dot{\alpha} \alpha} \psi_{\alpha} .
$$

A standard mass term contribution is given by employing the following Lagrangian

$$
\begin{aligned}
L_{0}+\frac{m}{2}\left(L_{m}+h . c .\right) & =A \partial^{2} \bar{A}+i \partial_{a} \bar{\psi}_{\dot{\alpha}} \bar{\sigma}^{a \dot{\alpha} \alpha} \psi_{\alpha}+F \bar{F} \\
& +m F A-\frac{1}{2} m \psi^{\alpha} \psi_{\alpha}+m \bar{F} \bar{A}-\frac{1}{2} m \bar{\psi}_{\dot{\alpha}} \bar{\psi}^{\dot{\alpha}} .
\end{aligned}
$$


A naive inspection of (1.4) would tell us that there is massive fermions, but no mass for the scalar fields has appeared. The equations of motion for the auxiliary field $F$ read

$$
\bar{F}=-m A
$$

and eventually, the on-shell form of (1.4) becomes

$$
L_{0}+\frac{m}{2}\left(L_{m}+\text { h.c. }\right)=A \partial^{2} \bar{A}+i \partial_{a} \bar{\psi}_{\dot{\alpha}} \bar{\sigma}^{a \dot{\alpha} \alpha} \psi_{\alpha}-m^{2} A \bar{A}-\frac{1}{2} m \psi^{\alpha} \psi_{\alpha}-\frac{1}{2} m \bar{\psi}_{\dot{\alpha}} \bar{\psi}^{\dot{\alpha}}
$$

where now we can see that supersymmetric masses have been raised. The lesson from the above discussion is that, until integrating out the auxiliary sector, it is not obvious if there exists a mass term, and in a more general context, what is the form of the scalar potential.

Turning to supergravity, the above discussion is straightforwardly generalised and the same prosedure is followed. The most general (two-derivative) superspace Lagrangian of chiral superfields coupled to supergravity is in superspace formalism ${ }^{1}$

$$
\mathscr{L}_{0}=\frac{1}{\kappa^{2}} \int d^{2} \Theta 2 \mathscr{E}\left[\frac{3}{8}(\overline{\mathscr{D}} \overline{\mathscr{D}}-8 \mathscr{R}) e^{-\frac{\kappa^{2}}{3} K\left(\Phi^{i}, \bar{\Phi}^{\bar{j}}\right)}+\kappa^{2} P(\Phi)\right]+\text { h.c. }
$$

The hermitian function $K\left(\Phi^{i}, \bar{\Phi}^{\bar{j}}\right)$ is the Kähler potential, $P\left(\Phi^{i}\right)$ is the superpotential (a holomorphic function of the chiral superfields $\Phi^{i}$ ) and $\kappa$ is proportional to the Planck length, which from now on will be set equal to 1 . From the supergravity multiplet sector, $2 \mathscr{E}$ is the usual chiral density employed to create supersymmetric Lagrangians, which in the new $\Theta$ variables has the expansion

$$
2 \mathscr{E}=e\left\{1+i \Theta \sigma^{a} \bar{\psi}_{a}-\Theta \Theta\left(M^{*}+\bar{\psi}_{a} \bar{\sigma}^{a b} \bar{\psi}_{b}\right)\right\}
$$

in terms of the vielbein $\left(e_{m}^{a}\right)$, the gravitino $\left(\psi_{m}\right)$ and the complex scalar auxiliary field $M$. In addition, $\mathscr{R}$, the superspace curvature, is a chiral superfield which contains the Ricci scalar in its highest component. In the matter sector, $\Phi^{i}$ and $\bar{\Phi}^{\bar{j}}$ denote a set on chiral and anti-chiral superfields $\left(\overline{\mathscr{D}}_{\dot{\alpha}} \Phi^{i}=0, \mathscr{D}_{\alpha} \bar{\Phi}^{\bar{j}}=0\right)$ whose components are defined via projection

$$
\begin{aligned}
A^{i} & =\left.\Phi^{i}\right|_{\theta=\bar{\theta}=0}, \\
\chi_{\alpha}^{i} & =\left.\frac{1}{\sqrt{2}} \mathscr{D}_{\alpha} \Phi^{i}\right|_{\theta=\bar{\theta}=0}, \\
F^{i} & =-\left.\frac{1}{4} \mathscr{D} \mathscr{D} \Phi^{i}\right|_{\theta=\bar{\theta}=0} .
\end{aligned}
$$

After calculating the component form of (1.7), integrating out the auxiliary fields and performing a Weyl rescaling of the gravitational field (accompanied by a redefinition of the fermionic fields), the pure bosonic Lagrangian reads

$$
e^{-1} \mathscr{L}_{0}=-\frac{1}{2} R-g_{i \bar{j}} \partial_{a} A^{i} \partial^{a} \bar{A}^{\bar{j}}-e^{K}\left[g^{i \bar{j}}\left(D_{i} P\right)\left(D_{\bar{j}} \bar{P}\right)-3 P \bar{P}\right] .
$$

Further details maybe found for example in [3]. Here

$$
g_{i \bar{j}}=\frac{\partial^{2} K(A, \bar{A})}{\partial A^{i} \partial \bar{A}^{\bar{r}}}
$$

\footnotetext{
${ }^{1}$ Our framework and conventions are those of Wess and Bagger [3].
} 
is the positive definite Kähler metric, on the manifold parametrized by $A^{i}$ and $\bar{A}^{\bar{j}}$. Moreover, the Kähler space covariant derivatives are defined as follows

$$
D_{i} P=P_{i}+K_{i} P
$$

where in general we denote $f_{i}=\frac{\partial f}{\partial A^{i}}$. The Lagrangian (1.10) is Kähler invariant as long as the superpotential scales as

$$
P\left(A^{i}\right) \rightarrow e^{-S\left(A^{i}\right)} P\left(A^{i}\right)
$$

under a Kähler transformation

$$
K\left(A^{i}, \bar{A}^{\bar{j}}\right) \rightarrow K\left(A^{i}, \bar{A}^{\bar{j}}\right)+S\left(A^{i}\right)+\bar{S}\left(\bar{A}^{\bar{j}}\right) .
$$

$S\left(A^{i}\right)$ and $\bar{S}\left(\bar{A}^{\bar{j}}\right)$ are holomorphic functions of the complex coordinates.

Equaly important conventional methods for introducing scalar potentials is by gauging the chiral models or by D-terms, the interested reader should consult [4].

\section{F-Emergent Potential}

The idea of the emergent potentials is essentialy a generalization of the standard methods discused above. The theory we are interested in, has a superspace Lagrangian of the form

$$
\mathscr{L}=\mathscr{L}_{0}+\mathscr{L}_{H D}
$$

where $\mathscr{L}_{0}$ is the standard superspace supergravity Lagrangian given in eq.(1.7) and [5, 6, 7]

$$
\mathscr{L}_{H D}=\int d^{2} \Theta 2 \mathscr{E}\left\{\frac{1}{8}(\overline{\mathscr{D}} \overline{\mathscr{D}}-8 \mathscr{R}) \Lambda^{\bar{r} i \bar{n} j}\left[\overline{\mathscr{D}}_{\dot{\alpha}} K_{i} \mathscr{D}_{\alpha} K_{\bar{r}} \overline{\mathscr{D}}^{\dot{\alpha}} K_{j} \mathscr{D}^{\alpha} K_{\bar{n}}\right]\right\}+\text { h.c. }
$$

This Lagrangian was initially studied in global supersymmetry in [8]. It is important that $\mathscr{L}$ is manifestly both Kähler and (independently) super-Weyl invariant as has been shown in [6]. These two symmetry properties, although obviously they do not specify the form of the action, they are essential in the consistency of the model as well as for the supergravity theory that it describes. As we will see, (2.2) does not involve derivatives of the auxiliary fields, which are not propagating and can be integrated out. Equivalently, (2.2) can be expressed in terms of the chiral superfields $\Phi^{i}$ as

$$
\mathscr{L}_{H D}=\int d^{2} \Theta 2 \mathscr{E}\left\{\frac{1}{8}(\overline{\mathscr{D}} \overline{\mathscr{D}}-8 \mathscr{R}) \Lambda_{i \bar{r} j \bar{n}}\left[\overline{\mathscr{D}}_{\dot{\alpha}} \bar{\Phi}^{\bar{r}} \mathscr{D}_{\alpha} \Phi^{i} \overline{\mathscr{D}}^{\dot{\alpha}} \bar{\Phi}^{\bar{n}} \mathscr{D}^{\alpha} \Phi^{j}\right]\right\}+\text { h.c. }
$$

where

$$
K_{i \bar{r}}=\frac{\partial^{2} K(\Phi, \bar{\Phi})}{\partial \Phi^{i} \partial \bar{\Phi}^{\bar{r}}}
$$

is the Kähler metric on the complex space spanned by the chiral and anti-chiral superfields and $\Lambda_{i \bar{r} j \bar{n}}$ represents a Kähler tensor. For example, one may choose

$$
\Lambda_{i \bar{r} j \bar{n}}=\mathscr{G}(\Phi, \bar{\Phi}) K_{i \bar{r}} K_{j \bar{n}}+\mathscr{H}(\Phi, \bar{\Phi}) \mathscr{R}_{i \bar{r} j \bar{n}}
$$


with $\mathscr{G}(\Phi, \bar{\Phi})$ and $\mathscr{H}(\Phi, \bar{\Phi})$ being some Kähler invariant hermitian functions and $\mathscr{R}_{i \bar{r} j \bar{n}}$ the Kähler space Riemann tensor defined as

$$
\mathscr{R}_{i \bar{j} k \bar{l}}=\frac{\partial}{\partial \Phi^{i}} \frac{\partial}{\partial \bar{\Phi}^{\bar{j}}} K_{k \bar{l}}-K^{m \bar{n}}\left(\frac{\partial}{\partial \bar{\Phi}^{\bar{j}}} K_{m \bar{l}}\right)\left(\frac{\partial}{\partial \Phi^{i}} K_{k \bar{n}}\right) .
$$

The form (2.5) implies some symmetries for the Kähler indices which, without loss of further generality, we will assume to be possessed by all the $\Lambda_{i \bar{r} j \bar{n}}$ to be considered in this work. Our next task is to extract the component field expression for the Lagrangian (2.3), which after superspace integration turns out to be

$$
\begin{aligned}
e^{-1} \mathscr{L}_{H D}=-16 \mathscr{U}_{i \bar{r} j \bar{n}} & \left(F^{i} F^{j} \bar{F}^{\bar{r}} \bar{F}^{\bar{n}}+\partial_{a} A^{i} \partial^{a} A^{j} \partial_{b} \bar{A}^{\bar{r}} \partial^{b} \bar{A}^{\bar{n}}\right. \\
& \left.-F^{i} \bar{F}^{\bar{r}} \partial_{a} A^{j} \partial^{a} \bar{A}^{\bar{n}}-F^{i} \bar{F}^{\bar{n}} \partial_{a} A^{j} \partial^{a} \bar{A}^{\bar{r}}\right) .
\end{aligned}
$$

for the pure bosonic sector. In (2.7) we have used the notation

$$
\mathscr{U}_{i \bar{r} j \bar{n}}(A, \bar{A})=\left.\Lambda_{i \bar{r} j \bar{n}}(\Phi, \bar{\Phi})\right|_{\theta=\bar{\theta}=0}
$$

Again it is easy to see that (2.7) is manifestly Kähler invariant.

In order to make the effect of the new coupling (2.2) more transparent we will consider now a theory with only one chiral multiplet and no superpotential. In this case, the Lagrangian (2.1) is explicitly written as

$$
\mathscr{L}=\int d^{2} \Theta 2 \mathscr{E}\left\{(\overline{\mathscr{D}} \overline{\mathscr{D}}-8 \mathscr{R})\left[\frac{3}{8} e^{-\frac{1}{3} K}+\frac{1}{8} \Lambda \overline{\mathscr{D}}_{\dot{\alpha}} \bar{\Phi} \mathscr{D} \alpha \Phi \overline{\mathscr{D}}^{\dot{\alpha}} \bar{\Phi}^{\alpha}{ }^{\alpha} \Phi\right]\right\}+\text { h.c. }
$$

with $\Lambda$ being an abbreviation for $\Lambda_{\Phi \Phi \Phi \bar{\Phi}}$, a hermitian and Kähler invariant function of $\Phi$ and $\bar{\Phi}$. In component form, the bosonic sector of the Lagrangian (2.9) turns out to be (after integrating out the auxiliary fields of the supergravity sector and subsequently appropriately rescaling)

$$
\begin{aligned}
e^{-1} \mathscr{L}_{\text {bos }}= & -\frac{1}{2} R-g_{A \bar{A}} \partial_{a} A \partial^{a} \bar{A}+g_{A \bar{A}} e^{\frac{K}{3}} F \bar{F} \\
& -16 \mathscr{U}\left\{e^{\frac{2 K}{3}}(F \bar{F})^{2}+\partial_{a} A \partial^{a} A \partial_{b} \bar{A} \partial^{b} \bar{A}-2 e^{\frac{K}{3}} F \bar{F} \partial_{a} A \partial^{a} \bar{A}\right\}
\end{aligned}
$$

where $\mathscr{U}$ is a hermitian Kähler invariant function of the scalar field (it is the lowest component of $\Lambda$, eq.(2.8)). The equation of motion for $F$ is

$$
\bar{F}\left(g_{A \bar{A}}-32 \mathscr{U} e^{\frac{K}{3}} F \bar{F}+32 \mathscr{U} \partial_{a} A \partial^{a} \bar{A}\right)=0
$$

which can be easily solved for

1. Standard solution:

$$
F=0,
$$

2. New solution:

$$
F \bar{F}=e^{\frac{-K}{3}}\left(\frac{g_{A \bar{A}}}{32 \mathscr{U}}+\partial_{a} A \partial^{a} \bar{A}\right) .
$$


Here we should discuss the difference between the two solutions. To make the point clear we first stress that the stability of the theory demands

$$
\begin{gathered}
g_{A \bar{A}}>0 \\
\mathscr{U}<0 .
\end{gathered}
$$

Thus the standard solution (2.12) can always be realized, while the new solution (2.13) can only be realized in the presence of fluxes so that

$$
F \bar{F}=e^{\frac{-K}{3}}\left(\frac{g_{A \bar{A}}}{32 \mathscr{U}}+\partial_{a} A \partial^{a} \bar{A}\right)>0 .
$$

The on-shell Lagrangian for the conventional branch is

$$
e^{-1} \mathscr{L}_{\text {bos }}=-\frac{1}{2} R-g_{A \bar{A}} \partial_{a} A \partial^{a} \bar{A}-16 \mathscr{U} \partial_{a} A \partial^{a} A \partial_{b} \bar{A} \partial^{b} \bar{A}
$$

where there is no scalar potential, as expected, since no superpotential was introduced. The on-shell Lagrangian for the new branch is

$$
e^{-1} \mathscr{L}_{\mathrm{bos}}=-\frac{1}{2} R+\frac{\left(g_{A \bar{A}}\right)^{2}}{64 \mathscr{U}}-16 \mathscr{U} \partial_{a} A \partial^{a} A \partial_{b} \bar{A} \partial^{b} \bar{A}+16 \mathscr{U} \partial_{a} A \partial^{a} \bar{A} \partial_{b} A \partial^{b} \bar{A}
$$

What has happened here has completely changed the dynamics of the theory. The minimal kinematic term for the scalar is lost and a scalar potential has emerged

$$
\mathscr{V}=-\frac{1}{64} \frac{\left(g_{A \bar{A}}\right)^{2}}{\mathscr{U}} .
$$

From (2.15) we see that the potential (2.19) is positive defined

$$
\mathscr{V}>0
$$

and therefore the theory may only have de Sitter vacua. Another important property of the emerging potential is that it is not built from a holomorphic function. Moreover, the function $\mathscr{U}$ governs now the kinetic terms and in fact it was shown in [5] that it has to be negative to avoid tachionic states. In the framework of new-minimal supergravity, consistent higher derivative terms which satsify the above restrictions have been considered [9], but no scalar potential emerged in that case.

\section{Gauge Invariant F-Emergent Potential}

The Lagrangian (2.9) can be straightforwardly be generalized to include gauge invariant interactions [10]. In this case, the gauge invariant superspace Lagrangian is

$$
\begin{aligned}
\mathscr{L}_{\text {tot }}=\int d^{2} \Theta 2 \mathscr{E}\{ & \frac{3}{8}(\overline{\mathscr{D}} \overline{\mathscr{D}}-8 \mathscr{R}) e^{-\tilde{K} / 3}+\frac{1}{16 g^{2}} \mathscr{H}_{(a b)}(\Phi) W^{(a)} W^{(b)}+P(\Phi) \\
& \left.+\frac{1}{8}(\overline{\mathscr{D}} \overline{\mathscr{D}}-8 \mathscr{R})\left[\tilde{\Lambda}^{\bar{r} i \bar{n} j} \overline{\mathscr{D}}_{\dot{\alpha}} \tilde{K}_{i} \mathscr{D}_{\alpha} \tilde{K}_{\bar{r}} \overline{\mathscr{D}}^{\dot{\alpha}} \tilde{K}_{j} \mathscr{D}^{\alpha} \tilde{K}_{\bar{n}}\right]\right\}+ \text { h.c. }
\end{aligned}
$$

where

$$
\tilde{K}=K(\Phi, \bar{\Phi})+\Gamma(\Phi, \bar{\Phi}, V)
$$


and

$$
\Gamma(\Phi, \bar{\Phi}, V)=V^{(a)} \mathscr{D}^{(a)}+\frac{1}{2} g_{i \bar{r}} X^{i(a)} \bar{X}^{\bar{r}(b)} V^{(a)} V^{(b)} .
$$

In addition, as usual, $V^{(a)}$ is the supersymmetric Yang-Mills vector multiplet and

$$
W_{\alpha}=W_{\alpha}^{(a)} T^{(a)}=-\frac{1}{4}(\overline{\mathscr{D}} \overline{\mathscr{D}}-8 \mathscr{R}) e^{-V} \mathscr{D}_{\alpha} e^{V}
$$

is the gauge invariant chiral superfield containing the gauge field strength. The holomorphic function $\mathscr{H}_{(a b)}$ is included for generality, but in what follows we will consider $\mathscr{H}_{(a b)}=\delta_{(a b)}$. Expression (3.3) is calculated in the Wess-Zumino gauge, $\mathscr{D}^{(a)}$ are the so-called Killing potentials whereas $X^{i(a)}$ and $\bar{X}^{\bar{r}(b)}$ are the components of the holomorphic Killing vectors that generate the isometries of the Kähler manifold. The Killing vectors and the Killing potential are connected via

$$
\begin{aligned}
g_{i \bar{r}} \bar{X}^{\bar{r}(a)} & =i \frac{\partial}{\partial a^{i}} \mathscr{D}^{(a)}, \\
g_{i \bar{r}} X^{i(a)} & =-i \frac{\partial}{\partial \bar{a}^{\bar{r}}} \mathscr{D}^{(a)}
\end{aligned}
$$

where $a^{i}$ and $\bar{a}^{\bar{r}}$ are the Kähler space complex co-ordinates. We note that the $\mathscr{D}^{(a)}$ that correspond to some $U(1)$ gauged symmetry are only determined up to a constant $\xi$, which is the analog for the Fayet-Iliopoulos D-term in supergravity. Now $\tilde{\Lambda}^{\bar{r} i \bar{n} j}$ has to respect all the isometries of the Kähler manifold. Again, following the standard procedure, the bosonic part of the Lagrangian (3.1) turns out to be

$$
\begin{aligned}
e^{-1} \mathscr{L}_{\text {tot }}= & -\frac{1}{2} R-g_{i \bar{r}} \tilde{D}_{m} A^{i} \tilde{D}^{m} \bar{A}^{\bar{r}}+e^{\frac{K}{3}} g_{i \bar{r}} F^{i} \bar{F}^{\bar{r}} \\
& -\frac{1}{16 g^{2}} F_{m n}^{(a)} F^{m n(a)}-\frac{1}{2} g^{2}\left(\mathscr{D}^{(a)}\right)^{2} \\
& -e^{\frac{2 K}{3}}\left(F^{i} D_{i} P+\bar{F}^{\bar{r}} D_{\bar{r}} \bar{P}\right)+3 e^{K} P \bar{P} \\
& -16 \tilde{\mathscr{U}}_{i \bar{r} j \bar{n}}\left(e^{\frac{2 K}{3}} F^{i} F^{j} \bar{F}^{\bar{r}} \bar{F}^{\bar{n}}+\tilde{D}_{a} A^{i} \tilde{D}^{a} A^{j} \tilde{D}_{b} \bar{A}^{\bar{r}} \tilde{D}^{b} \bar{A}^{\bar{n}}\right. \\
& \left.-e^{\frac{K}{3}} F^{i} \bar{F}^{\bar{r}} \tilde{D}_{a} A^{j} \tilde{D}^{a} \bar{A}^{\bar{n}}-e^{\frac{K}{3}} F^{i} \bar{F}^{\bar{n}} \tilde{D}_{a} A^{j} \tilde{D}^{a} \bar{A}^{\bar{r}}\right) .
\end{aligned}
$$

We note that

$$
\tilde{D}_{c} A^{j}=\partial_{c} A^{j}-\frac{1}{2} B_{c}^{(a)} X_{(a)}^{j}
$$

is the covariant derivative and $B_{c}^{(a)}$ is a vector field (belonging to the $V^{(a)}$ vector multiplet) that corresponds to the gauged isometries, with field strength $F_{m n}^{(a)}$.

In order to illustrate the properties of the emergent potential in the case of gauged models, our example will be a single chiral multiplet with no superpotential. In this case the Lagrangian (3.7) is

$$
\begin{aligned}
e^{-1} \mathscr{L}_{t o t}= & -\frac{1}{2} R-g_{A \bar{A}} \tilde{D}_{m} A \tilde{D}^{m} \bar{A}+e^{\frac{K}{3}} g_{A \bar{A}} F \bar{F} \\
& -\frac{1}{16 g^{2}} F_{m n}^{(a)} F^{m n(a)}-\frac{1}{2} g^{2}(\mathscr{D}(a))^{2} \\
& -16 \tilde{\mathscr{U}}\left(e^{\frac{2 K}{3}}(F \bar{F})^{2}+\tilde{D}_{a} A \tilde{D}^{a} A \tilde{D}_{b} \bar{A} \tilde{D}^{b} \bar{A}-2 e^{\frac{K}{3}} F \bar{F} \tilde{D}_{a} A \tilde{D}^{a} \bar{A}\right) .
\end{aligned}
$$


The single auxiliary field $F$ can now be eliminated from (3.9) by its equations of motion, leading to

$$
F \bar{F}=e^{\frac{-K}{3}}\left(\frac{g_{A \bar{A}}}{32 \tilde{\mathscr{U}}}+\tilde{D}_{a} A \tilde{D}^{a} \bar{A}\right)
$$

Plugging (3.10) back in (3.9), we can easily read-off the potential for the gauged model which turns out to be

$$
\mathscr{V}=\frac{1}{2} g^{2}\left(\mathscr{D}^{(a)}\right)^{2}-\frac{\left(g_{A \bar{A}}\right)^{2}}{64 \tilde{\mathscr{U}}}
$$

with $\tilde{\mathscr{U}}=\tilde{\mathscr{U}}_{A \bar{A} A \bar{A}}$, a Kähler-space tensor that respects all the isometries of the gauged group. For a first example we will take a flat model with Kähler potential

$$
K=a \bar{a}+d
$$

which leads to

$$
g_{a \bar{a}}=1, \quad \mathscr{R}_{a \bar{a} a \bar{a}}=0
$$

The $U(1)$ Killing potential is

$$
D^{(1)}=a \bar{a}+\xi
$$

where the parameter $\xi$ corresponds to the aforementioned freedom to shift the $U(1)$ Killing potential. When we promote $a$ and $\bar{a}$ to the superfields $\Phi$ and $\bar{\Phi}$, our Kähler potential $K$ together with the counter term $\Gamma$ become

$$
\tilde{K}_{U(1)}=\Phi \bar{\Phi}+V \Phi \bar{\Phi}+\frac{1}{2} V^{2} \Phi \bar{\Phi}+d+V \xi
$$

The bosonic part of our Lagrangian in component form then turns out to be

$$
\begin{aligned}
e^{-1} \mathscr{L}_{U(1)}= & -\frac{1}{2} R-\frac{1}{16 g^{2}} F_{c d} F^{c d} \\
& -16 \tilde{\mathscr{U}} \tilde{D}_{a} A \tilde{D}^{a} A \tilde{D}_{b} \bar{A} \tilde{D}^{b} \bar{A}+16 \tilde{\mathscr{U}} \tilde{D}_{a} A \tilde{D}^{a} \bar{A} \tilde{D}_{b} A \tilde{D}^{b} \bar{A} \\
& -\frac{1}{2} g^{2}(A \bar{A}+\xi)^{2}+\frac{1}{64 \tilde{\mathscr{U}}},
\end{aligned}
$$

with $\tilde{D}_{m} A=\partial_{m} A+\frac{i}{2} B_{m} A$. Then the scalar potential is

$$
\mathscr{V}=\frac{1}{2} g^{2}\left(D^{(a)}\right)^{2}-\frac{1}{64 \tilde{\mathscr{U}}} .
$$

A simple choice for $\tilde{\mathscr{U}}$ could be

$$
\tilde{\mathscr{U}}=m g_{A \bar{A}} g_{A \bar{A}}=m<0,
$$

where $m$ is a negative constant. It is again important to emphasise that $m$ now governs the kinematics of the scalar fields, and that the condition

$$
F \bar{F}=e^{\frac{-K}{3}}\left(\frac{g_{A \bar{A}}}{32 \tilde{\mathscr{U}}}+\tilde{D}_{a} A \tilde{D}^{a} \bar{A}\right)>0
$$

has to hold for the theory to be consistent. 


\section{D-Emergent Potential}

Higher derivative interactions are not restricted only to scalar fields. In fact we will show that an equivalent method as before can be followed which again leads to a scalar potential. Now the auxiliary fields that are integrated out are the ones of the vector multiplet, the " $D$ " fields.

The higher derivative term we want to discuss is (in superspace)

$$
\mathscr{L}_{g H D}=\int d^{2} \Theta 2 \mathscr{E}(\overline{\mathscr{D}} \overline{\mathscr{D}}-8 \mathscr{R})\left(-\frac{1}{4} \mathscr{J}_{a b}(\Phi, \bar{\Phi}) W^{(a)} W^{(b)} \mathscr{Y}_{c d}(\Phi, \bar{\Phi}) \bar{W}^{(c)} \bar{W}^{(d)}\right)+\text { h.c. }
$$

The superfields $\mathscr{J}_{a b}(\Phi, \bar{\Phi})$ and $\mathscr{Y}_{c d}(\Phi, \bar{\Phi})$ are functions of the various chiral superfields that are present in our theory, the only restriction is that they should transform correctly under the gauge group. The bosonic sector of Lagrangian (4.1) after performing the superspace integration is

$$
\begin{aligned}
e^{-1} \mathscr{L}_{g H D}= & {\left[J_{a b} \bar{Y}_{c d}+\bar{J}_{a b} Y_{c d}\right] \times } \\
\{ & \frac{1}{4} F^{d c(a)} F_{d c}^{(b)} F^{a b(c)} F_{a b}^{(d)}-\frac{1}{2} F^{d c(a)} F_{d c}^{(b)} D^{(c)} D^{(d)}-\frac{1}{2} D^{(a)} D^{(b)} F^{a b(c)} F_{a b}^{(d)} \\
& \left.+D^{(a)} D^{(b)} D^{(c)} D^{(d)}+\frac{1}{16} \varepsilon^{a b c d} F_{a b}^{(a)} F_{c d}^{(b)} \varepsilon^{e f g h} F_{e f}^{(c)} F_{g h}^{(d)}\right\} .
\end{aligned}
$$

Here $J_{a b}=\mathscr{J}_{a b} \mid$ and $Y_{a b}=\mathscr{Y}_{a b} \mid$. Moreover for the gauge sector we will consider a more general coupling allowing for a kinetic gauge function as well. The standard kinetic term for the gauge fields is

$$
\mathscr{L}_{g 0}=\int d^{2} \Theta 2 \mathscr{E} \mathscr{H}_{(a b)}(\Phi) W^{(a)} W^{(b)}+\text { h.c. }
$$

and the bosonic sector in components reads

$$
e^{-1} \mathscr{L}_{g 0}=\left[H_{(a b)}+\bar{H}_{(a b)}\right]\left\{-\frac{1}{2} F^{d c(a)} F_{d c}^{(b)}-\frac{i}{4} \varepsilon^{a b c d} F_{a b}^{(a)} F_{c d}^{(b)}+D^{(a)} D^{(b)}\right\}
$$

with $H_{a b}=\mathscr{H}_{a b} \mid$. Up to now the most general Lagrangian in superspace reads

$$
\begin{aligned}
\mathscr{L}_{\text {tot }}=\int d^{2} \Theta 2 \mathscr{E}\{ & \frac{3}{8}(\overline{\mathscr{D}} \overline{\mathscr{D}}-8 \mathscr{R}) e^{-\tilde{K} / 3}+\mathscr{H}_{(a b)}(\Phi) W^{(a)} W^{(b)}+P(\Phi) \\
& +\frac{1}{8}(\overline{\mathscr{D}} \overline{\mathscr{D}}-8 \mathscr{R})\left[\tilde{\Lambda}^{\bar{r} i \bar{n} j} \overline{\mathscr{D}}_{\dot{\alpha}} \tilde{K}_{i} \mathscr{D}_{\alpha} \tilde{K}_{\bar{r}} \overline{\mathscr{D}}^{\dot{\alpha}} \tilde{K}_{j} \mathscr{D}^{\alpha} \tilde{K}_{\bar{n}}\right] \\
& \left.-\frac{1}{4}(\overline{\mathscr{D}} \overline{\mathscr{D}}-8 \mathscr{R})\left[\mathscr{J}_{a b}(\Phi, \bar{\Phi}) W^{(a)} W^{(b)} \mathscr{Y}_{c d}(\Phi, \bar{\Phi}) \bar{W}^{(c)} \bar{W}^{(d)}\right]\right\}+ \text { h.c. }
\end{aligned}
$$

Finally, in order to study the properties of this new term, let us consider a very simple example of a single $U(1)$ group and a single uncharged (under this $U(1)$ ) chiral multiplet. The higher derivative terms will be only for the gauge sector. Our Lagrangian, in component form reads

$$
\begin{aligned}
e^{-1} \mathscr{L}_{e x}= & -\frac{1}{2} R-g_{A \bar{A}} \partial_{m} A \partial^{m} \bar{A}+[H(A)+\bar{H}(\bar{A})]\left\{-\frac{1}{2} F^{d c} F_{d c}-\frac{i}{4} \varepsilon^{a b c d} F_{a b} F_{c d}+D^{2}\right\} \\
& +[J \bar{Y}+Y \bar{J}]\left\{\frac{1}{4}\left(F^{d c} F_{d c}\right)^{2}-F^{d c} F_{d c} D^{2}+\frac{1}{16}\left(\varepsilon^{a b c d} F_{a b} F_{c d}\right)^{2}+D^{4}\right\} .
\end{aligned}
$$

Here $J$ and $Y$ are positive definite gauge invariant functions of $A$ and $\bar{A}$. Now we can easily solve the auxiliary $D$ equations of motion to find two solutions 
1. Standard solution:

$$
D=0,
$$

2. New solution:

$$
D^{2}=\frac{1}{2} F^{d c} F_{d c}-\frac{1}{2} \frac{H+\bar{H}}{J \bar{Y}+Y \bar{J}} .
$$

The first one is the standard supersymmetric solution and has been also studied in [1] in the presence of higher derivatives. The new solution can only be consistently realized in the presence of magnetic fluxes so that

$$
D^{2}=\frac{1}{2} F^{d c} F_{d c}-\frac{1}{2} \frac{H+\bar{H}}{J \bar{Y}+Y \bar{J}}>0 .
$$

Eventually the on-shell theory will be

$$
\begin{aligned}
e^{-1} \mathscr{L}_{e x}= & -\frac{1}{2} R-g_{A \bar{A}} \partial_{m} A \partial^{m} \bar{A}-\frac{1}{4} \frac{(H+\bar{H})^{2}}{J \bar{Y}+Y \bar{J}}-\frac{i}{4}[H(A)+\bar{H}(\bar{A})] \varepsilon^{a b c d} F_{a b} F_{c d} \\
& +\frac{1}{16}[J \bar{Y}+Y \bar{J}]\left(\varepsilon^{a b c d} F_{a b} F_{c d}\right)^{2} \\
= & -\frac{1}{2} R-g_{A \bar{A}} \partial_{m} A \partial^{m} \bar{A}-\frac{1}{4} \frac{(H+\bar{H})^{2}}{J \bar{Y}+Y \bar{J}}-\frac{i}{4}[H(A)+\bar{H}(\bar{A})] \varepsilon^{a b c d} F_{a b} F_{c d} \\
& +[J \bar{Y}+Y \bar{J}]\left\{-\frac{1}{2}\left(F^{d c} F_{d c}\right)^{2}+F_{a b} F^{b c} F_{c d} F^{d a}\right\} .
\end{aligned}
$$

It is easy to see that there is a positive definite emergent potential due to integrating out of the $D$ auxiliary field

$$
\mathscr{V}(A, \bar{A})=\frac{1}{4} \frac{(H+\bar{H})^{2}}{J \bar{Y}+Y \bar{J}} .
$$

A simple example can be given by a gauge kinetic function

$$
H=A^{2}
$$

with $a, b$ two real positive constants

$$
J=a>0, \quad Y=b>0 .
$$

The potential will be

$$
\mathscr{V}(A, \bar{A})=\frac{\left(A^{2}+\bar{A}^{2}\right)^{2}}{8 a b} .
$$

This novel feature of gauge fields higher derivatives has not been studied before and deserves further investigation.

Summarizing, the well-known standard form of the $\mathrm{N}=1$ scalar potential is restricted to the two-derivative level. Higher derivative interaction modify its form. In fact, when higher-derivatives are introduced, an emerging scalar potential appears even if there is no superpotential to start with. There are two types of emerging potential, F- and D-type. F-emerging potentials result by integrating out auxiliaries of chiral multiplets whereas, D-emerging potentials come from the integration of auxiliaries in vector multiplets. As a general rule, emerging potentials are positive defined with de Sitter ground state, indicating supersymmetry breaking. 


\section{References}

[1] S. Cecotti, S. Ferrara and L. Girardello, "Structure Of The Scalar Potential In General N=1 Higher Derivative Supergravity In Four-dimensions,” Phys. Lett. B 187, 321 (1987).

[2] M. Ostrogradski, 1850 Memoires sur les equations differentielles relatives au probleme des isoperimetres, Mem. Ac. St. Petersburg, VI Series, Vol. 4385.

[3] J. Wess and J. Bagger, "Supersymmetry and supergravity," Princeton, USA: Univ. Pr. (1992) $259 \mathrm{p}$

[4] G. Villadoro and F. Zwirner, “De-Sitter vacua via consistent D-terms,” Phys. Rev. Lett. 95, 231602 (2005) [hep-th/0508167].

[5] M. Koehn, J. -L. Lehners and B. A. Ovrut, "Higher-Derivative Chiral Superfield Actions Coupled to N=1 Supergravity,” Phys. Rev. D 86, 085019 (2012) [arXiv:1207.3798 [hep-th]].

[6] F. Farakos and A. Kehagias, "Emerging Potentials in Higher-Derivative Gauged Chiral Models Coupled to N=1 Supergravity,” JHEP 1211, 077 (2012) [arXiv:1207.4767 [hep-th]].

[7] M. Koehn, J. -L. Lehners and B. A. Ovrut, “DBI Inflation in N=1 Supergravity,” Phys. Rev. D 86, 123510 (2012) [arXiv:1208.0752 [hep-th]].

[8] J. Khoury, J. -L. Lehners and B. Ovrut, "Supersymmetric P(X, $\phi)$ and the Ghost Condensate," Phys. Rev. D 83, 125031 (2011) [arXiv:1012.3748 [hep-th]].

[9] F. Farakos, C. Germani, A. Kehagias and E. N. Saridakis, "A New Class of Four-Dimensional N=1 Supergravity with Non-minimal Derivative Couplings," JHEP 1205, 050 (2012) arXiv:1202.3780 [hep-th].

[10] J. A. Bagger, "Coupling the Gauge Invariant Supersymmetric Nonlinear Sigma Model to Supergravity,” Nucl. Phys. B 211, 302 (1983). 